\title{
The Atoms of Matter; their Size, Number, and Construction. ${ }^{1}$ \\ By Dr. F. W. Aston, F.R.S.
}

THA

HAT matter is discontinuous and consists of discrete particles is now an accepted fact, but it is by no means obvious to the senses. The surfaces of clean liquids, even under the most powerful micro-

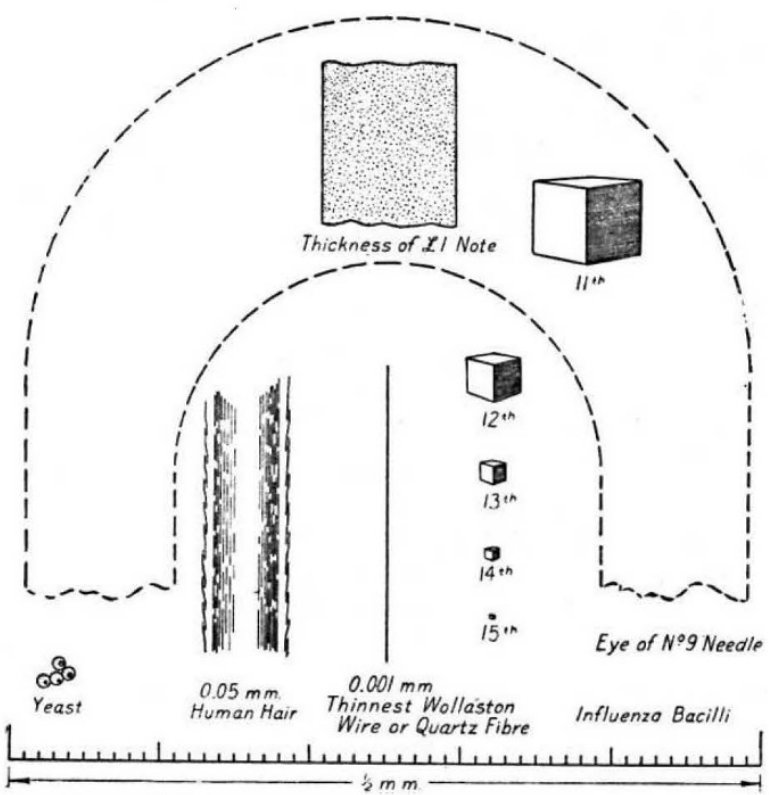

FIG. x.-Cubes II to 15 compared with familiar objects to scale.

scope, appear perfectly smooth, coherent, and continuous. The merest trace of a soluble dye will colour millions of times its volume of water. It is not surprising, therefore, that in the past there have arisen schools which believed that matter was quite continuous and infinitely divisible.

The upholders of this view said that if you took a piece of material, lead, for example, and went on cutting it into smaller and smaller fragments with a sufficiently sharp knife, you could go on indefinitely. The opposing school argued that at some stage in the operations either the act of section would become impossible, or the result would be lead no longer. Bacon, Descartes, Gassendi, Boyle, and Hooke were all partial to the latter theory, and Newton in 1675 tried to explain Boyle's Law on the assumption that gases were made up of mutually repulsive particles.

The accuracy of modern knowledge is such that we can carry out, indirectly at least, the experiment suggested by the old philosophers right up to the stage when the second school is proved correct, and the ultimate atom of lead reached. For convenience, we will start with a standard decimetre cube of lead weighing $1 \mathrm{I} \cdot 37$ kilograms, and the operation of section will consist of three cuts at right angles to each other, dividing the original cube into eight similar bodies each of half the linear dimensions and one-eighth the weight. Thus the first cube will have $5 \mathrm{~cm}$. sides and weigh $x \cdot 42$ kilograms, the second will weigh $178 \mathrm{gm}$., the fourth $2.78 \mathrm{gm}$., and so on. Diminution in the ${ }^{1}$ From an evening discourse delivered before the British Association at
Hull, September 12,1922 . series is very rapid and the result of the ninth operation is a quantity of lead just weighable on the ordinary chemical balance. The results of further operations are compared with suitable objects and a scale of length in Figs. I, 2, and 3. The last operation possible, without breaking up the lead atom, is the twentyeighth. The twenty-sixth cube is illustrated in Fig. 3. It contains 64 atoms, the size, distance apart, and general arrangement of which can be represented with considerable accuracy, thanks to the exact knowledge derived from research on X-rays and specific heats. On the same scale are represented the largest atom, cæsium, and the smallest atom, carbon, together with molecules of oxygen and nitrogen, at their average distance apart in the air, and the helical arrangement of silicon and oxygen atoms in quartz crystals discovered by X-ray analysis. The following table shows at what stages certain analytical methods break down. The great superiority of the microscope is a noteworthy point.

$\begin{array}{rlcl}\text { Cube. } & \text { Side in Cm. } & \text { Mass in Gm. } & \text { Limiting Analytical Method. } \\ 9 & 0.0195 & 8.5 \times 10^{-5} & \text { Ordinary Chemical Balance } \\ \text { 14 } & 6.1 \times 10^{-4} & 2.58 \times 10^{-9} & \text { Quartz Micro-balance } \\ \text { I5 } & 3.05 \times 10^{-4} & 3.22 \times 10^{-10} & \text { Spectrum Analysis (Na lines) } \\ \text { 18 } & 3.8 \times 10^{-5} & 6.25 \times 10^{-13} & \text { Ordinary Microscope } \\ 24 & 6.0 \times 10^{-7} & 2.38 \times 10^{-18} & \text { Ultra Microscope } \\ 28 & 3.7 \times 10^{-8} & 5.15 \times 10^{-22} & \\ \text { Atom. } & 3.0 \times 10^{-8} & 3.44 \times 10^{-32} & \text { Radioactivity }\end{array}$

Just as any vivid notion of the size of the cubes passes out of our power at about the twelfth-the limiting size of a dark object visible to the unaided eye-so when one considers the figures expressing the number of atoms in any ordinary mass of material, the mind is staggered by their immensity. Thus if

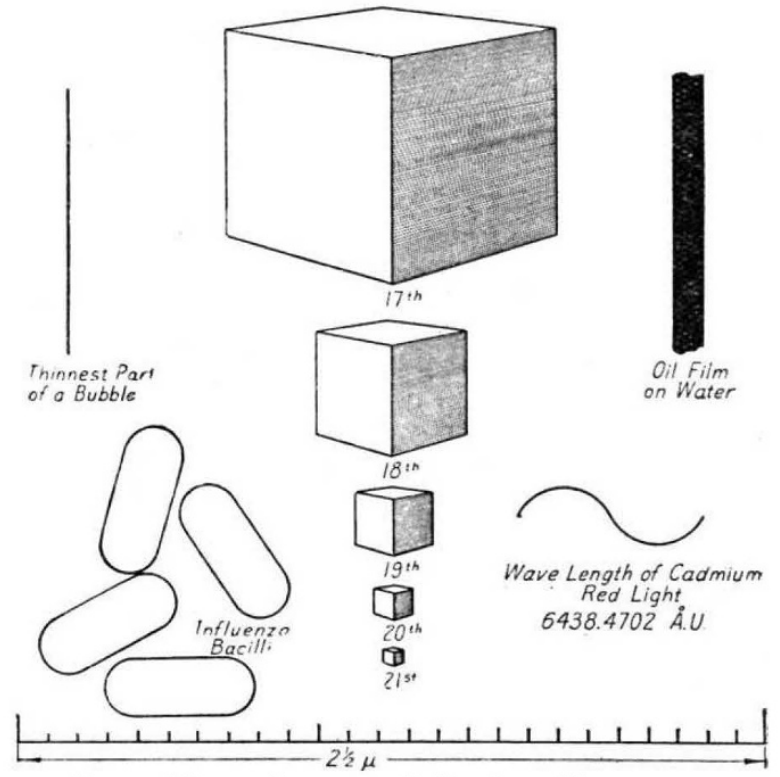

FIG. 2.-Cubes I7 to 2 I compared with minute objects to scale.

we slice the original decimetre cube into square plates one atom thick the area of these plates will total one and one-quarter square miles. If we cut these plates into strings of atoms spaced apart as they are in the 
solid, these decimetre strings put end-to-end will reach 6.3 million million miles, the distance light will travel in a year, a quarter of the distance to the nearest fixed star. If the atoms are spaced but one millimetre apart the string will be three and a half million times longer yet, spanning the whole universe.

Again, if an ordinary evacuated electric light bulb were pierced with an aperture such that one million molecules of the air entered per second, the pressure in the bulb would not rise to that of the air outside for a hundred million years. Perhaps the most striking illustration is as follows: Take a tumbler of water and-supposing it possible-label all the molecules in it. Throw the water into the sea, or, indeed, anywhere you please, and after a period of time so great that all the water on the earth-in seas, lakes, rivers, and clouds-has had time to become perfectly mixed, fill your tumbler again at the nearest tap. How many of the labelled molecules are to be expected in it? The answer is, roughly, 2000; for although the number of tumblerfuls of water on the earth is $5 \times 10^{21}$, the number of molecules of water in a single tumbler is $10^{25}$.

From the above statements it would, at first sight, appear absurd to hope to obtain effects from single atoms, yet this can now be done in several ways, and indeed it is largely due to the results of such experiments that the figures can be stated with so much confidence. Detection of an individual is only feasible in the case of an atom moving with an enormous velocity when, although its mass is so minute, its energy is quite appreciable. The charged helium atom shot out by radioactive substances in the form of an alpha ray possesses so much energy that the splash of light caused by its impact against a fluorescent screen can be visibly detected, the ionisation caused by its passage through a suitable gas can be measured on a sensitive electrometer and, in the beautiful experiments of C. T. R. Wilson, its path in air can be seen and photographed by means of the condensation of water drops upon the atomic wreckage it leaves behind it.

In the first complete Atomic Theory put forward by Dalton in 1803 one of the postulates states that: "Atoms of the same element are similar to one another and equal in weight." Of course, if we take this as a definition of the word "element" it becomes a truism, but, on the other hand, what Dalton probably meant by an element, and what we understand by the word to-day, is a substance such as hydrogen, oxygen, chlorine, or lead, which has unique chemical properties and cannot be resolved into more elementary constituents by any known chemical process. For many of the well-known elements Dalton's postulate still appears to be strictly true, but for the others, probably the majority, it needs some modification.

Throughout the history of science philosophers have been in favour of the idea that all matter is composed of the same primordial substance, and that the atoms of the elements are simply stable aggregations of atoms of this substance. Shortly after Dalton's theory had been put forward Prout suggested that the atoms of the elements were composed of atoms of a substance he called "protyle," which he endeavoured to identify with hydrogen.

If Dalton and Prout were both right the combining weights of the elements should all be expressible as whole numbers, hydrogen being unity. Experimental evidence showed this to be impossible in many cases. Chemists therefore wisely preferred Dalton's theory, which was in accord with definite though fractional atomic weights, to Prout's, which would necessitate the elements of fractional atomic weight being heterogeneous mixtures of atoms of different weight.

The idea that atoms of the same element are all identical in weight could not be challenged by ordinary chemical methods, for the atoms are by definition chemically identical, and numerical ratios were only to be obtained in such methods by the use of quantities of the element containing countless myriads of atoms. At the same time it is rather surprising, when we consider the complete absence of positive evidence in its support, that no theoretical doubts were publicly expressed until late in the nineteenth century, first by

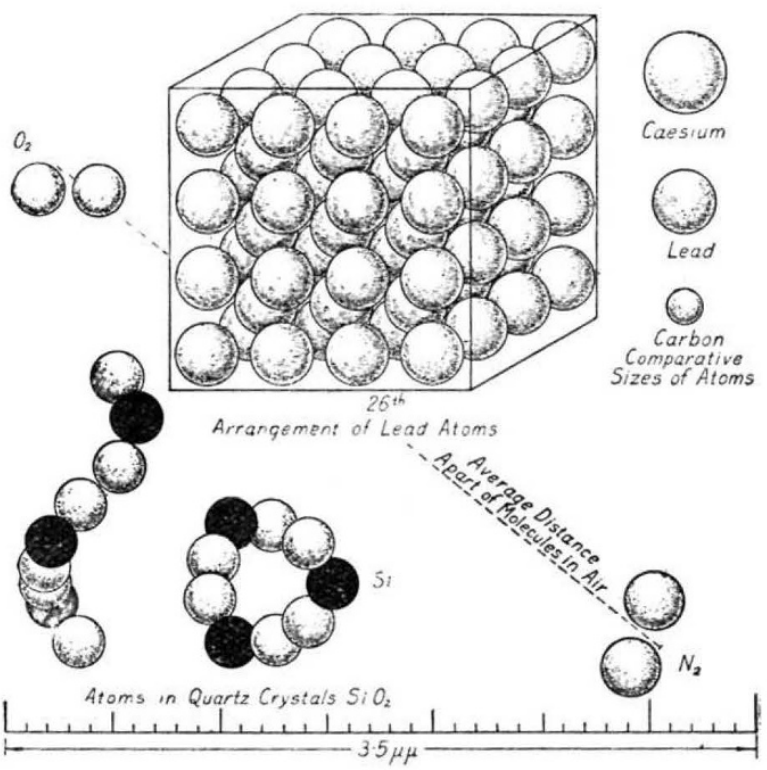

Fic. 3.-Cube 26 showing atoms with scale of reference.

Schutzenberger and then by Crookes, and that these doubts have been regarded, even up to the last few years, as speculative in the highest degree. In order to dismiss the idea that the atoms of such a familiar element as chlorine might not all be of the same weight, one had only to mention diffusion experiments and the constancy of chemical equivalents. It is only within the last few years that the lamentable weakness of such arguments has been exposed and it has been realised that the experimental separation of atoms differing from each other by so much as ro per cent. in weight, is really an excessively difficult operation.

There are two ways by which the identity of the weights of the atoms forming an element can be tested. One is by the direct comparison of the weights of individual atoms; the other is by obtaining samples of the element from different sources or by different processes, which, although perfectly pure, do not give the same chemical atomic weight. It was by the second and less direct of these methods that it was first shown by the experiments of Soddy and others on the atomic weight of lead from different radioactive 
sources, that substances could exist which, though chemically identical, had different atomic weights. These substances Soddy called "isotopes" as they occupy the same place in the periodic table of the elements.

The first experimental comparison of the weights of individual atoms was made by Sir J. J. Thomson in his analysis of positive rays by the " parabola " method. Subjected to this test most of the lighter elements appeared to follow Dalton's rule, but the results with the rare gas neon suggested the possibility of the atoms of this element being of two different weights, roughly 20 and $\mathbf{2 2}$ respectively. In other words the parabolas of neon indicated that it might be a mixture of isotopes, but the accuracy of measurement by this method was not sufficient to settle the point with certainty.

The requisite accuracy has been obtained by an instrument for the analysis of positive rays called the "mass-spectrograph." By this device, the weights of atoms can be compared to an accuracy of onetenth per cent., and it has been demonstrated not only that neon $(\mathbf{2 0 \cdot 2 )}$ is a mixture of atoms of weights exactly 20 and 22 , but also that chlorine $\left(35^{\circ} 46\right)$ is a mixture of isotopic atoms of weights 35 and 37 . Furthermore, about half the elements investigated turn out to be mixtures, some of the heavier ones consisting of six or more different constituents. Most important of all is the fact that every element investigated, with the exception of hydrogen, consists of atoms the weights of which are expressible as whole numbers on the oxygen scale used by chemists.

This remarkable generalisation called the "whole number rule" has removed the last obstacle in the way of the unitary theory of matter. We now have no hesitation in affirming that Nature uses the same standard bricks in the construction of the atoms of all elements, and that these standard bricks are the primordial atoms of positive and negative electricity, protons and electrons.

These are the natural unit charges of electricity, equal but of opposite sign. Of the shape of these particles we know next to nothing, but the wonderful advances of modern physics, in particular those of radioactivity, enable us to speak of their weights and dimensions with some assurance. The weight of the proton is very nearly the weight of a hydrogen atom, the electron is nearly two thousand times lighter, so that the atomic weight of an element (not consisting of isotopes) will be roughly equal to the number of protons in its atoms. The dimensions of the electron are about one hundred thousand times less than those of the atoms as illustrated above, and the proton is probably nearly two thousand times smaller still.

We now know of what atoms are constructed, and may go on to consider the evidence as to how their constituent parts are arranged. In the foregoing diagrams the atoms are represented as spheres, and in respect to the small forces and velocities which occur in the collisions between the atoms of gases at ordinary temperatures they do behave very exactly as smooth elastic spheres. But unfortunately the idea of a sphere carries the suggestion of a portion of space full of something ; that is, the atom as a sort of spherical bag packed full of electric charges. Nothing could be further from the actuality, for from the figures already given, it can be seen at once that even in the heaviest atom known the constituent charges fail to fill even the million millionth part of its whole volume. To convey any direct idea of these numerical relations by diagrams is practically hopeless, and were we to construct a scale model of the atom as big as the dome of St. Paul's we should have some difficulty in seeing the electrons, which would be little larger than pin heads, while the protons would escape notice altogether as dust particles invisible to the unaided eye. Experimental evidence leaves us no escape from the astounding conclusion that the atom of matter, as a structure, is empty, empty as the solar system, and what we measure as its spherical boundary really only represents the limiting orbits of its outermost electrons.

The hypothesis which has led to the greatest advances in our knowledge of the inner construction of atoms is Rutherford's theory of the "nucleus atom" put forward in I9II. This is supported by so many results of direct experiment that it is now universally accepted and must be substantially correct. It postulates that all of the positive and about half of the negative electricity, that is, practically the entire weight, of the atom is concentrated at its centre, forming a very small body called the nucleus. In other words, all the protons and about half the electrons in the atom are packed together, forming a sort of sun round which revolve the remaining electrons as planets. The number of protons in excess of electrons in the nucleus will clearly be its net positive charge, and since this will not depend on the gross numbers of protons and electrons but only on their difference, we can have elements the atoms of which have nuclei of different weights but the same net charge. These are isotopes, for the chemical properties of an atom are determined by the charge on its nucleus.

The nucleus is extremely small compared with the whole atom. Thus, if in the atom of helium atomic weight 4 atomic number 2 we take the nucleus, consisting of 4 protons and 2 electrons, as represented by a rather large pea, its planetary electrons may be represented on the same scale as two rather smaller peas revolving round it at a distance of a quarter of $a$ mile. The dislodgement of one of its planetary electrons from an atom requires comparatively little energy and is the well-known process called ionisation. This change is only a temporary one, as the atom takes the first opportunity of attracting it or any other stray electron back into its orbit and becoming neutral again. It is by a sort of continual exchange of such loose electrons that electricity is conducted along metallic wires. Disruption of the nucleus, on the other hand, needs enormous energy, but once performed must give rise to the atom of a new element. This process of transmutation has been achieved by Sir Ernest Rutherford, in the case of some of the lighter elements, by bombarding their atoms with alpha rays, which are charged helium nuclei expelled at enormous speeds from radioactive atoms during their natural process of disintegration. From the tiny dimensions of the nucleus compared with those of the atom it is obvious that the chance of getting a direct hit on the nucleus is only one in many millions, but the experiments show that when this does take place 
protons are dislodged from the atoms of the element struck and that therefore transmutation has been actually carried out.

The quantity of matter so transmitted is indeed almost inconceivably small, but it is the first step towards what may well be the greatest achievement of the human race, the release and control of the socalled "atomic energy." We now know with certainty that four neutral hydrogen atoms weigh appreciably more than one neutral helium atom, though they contain identically the same units, 4 protons and 4 electrons. The change of weight is probably due to the closer "packing" in the helium nucleus, but whatever the explanation may be transmutation of hydrogen into helium must inevitably destroy matter and therefore liberate energy. The quantity of energy can be calculated and is prodigious beyond the dreams of scientific fiction. If we could transmute the hydrogen contained in one pint of water the energy so liberated would be sufficient to propel the Mauretania across the Atlantic and back at full speed. With such vast stores of energy at our disposal there would be literally no limit to the material achievements of the human race.

The possibility that the process of transmutation might be beyond control and result in the detonation of all the water on the earth at once is an interesting one, since, in that case, the earth and its inhabitants would be dissipated into space as a new star, but the probability of such a catastrophe is too remote to be considered seriously. A recent newspaper article pointed out the danger of scientific discovery, and actually suggested that any results of research which might lead to the liberation of atomic energy should be suppressed. So, doubtless, the more elderly and apelike of our prehistoric ancestors grumbled at the innovation of cooked food, and gravely pointed out the terrible dangers of the newly-invented agency, fire, but it can scarcely be maintained to-day that subsequent history has justified their caution.

\section{The Herring Fishery and its Fluctuations.}

By B. Storrow, Dove Marine Laboratory, Cullercoats, Northumberland.

$\mathrm{H}^{2}$ ERRINGS are fished in every month of the year, and the catches show considerable variation in the size of the fish, the state of the reproductive organs, and the age composition of the shoals. It is necessary, therefore, before arriving at any conclusion with regard to the fishery, to take into consideration the kinds of herrings which are caught on the different grounds throughout the year.

In the beginning of the year, January, February, and March, shoals are fished about the north-west of Ireland, off the north of Scotland, including the Shetlands and Orkneys, and in the Firth of Forth. These herrings are all fish with the gonads well developed, and they spawn towards the end of February or in March. They are known as spring spawners and, except for the shoals of the Firth of Forth, they, so far as the western part of the North Sea is concerned, are caught in northern waters. In April the spent fish from the spring spawning shoals are caught all over the North Sea, from the Shetlands to Bergen Bank, from North Shields to the Naze, and off Yarmouth and Lowestoft. The catches are used chiefly for bait by the drift-net fishermen, who at this time are fishing with lines for cod, ling, halibut, etc. Among some of the bait catches are found numbers of small fish with the gonads not developed, and without doubt these can be classified as virgin fish.

During May the number of drifter-liners decreases and catches of herrings are made from ten to thirty miles off our coast. These catches consist of young fish with the gonads at practically the same stage of development as those found in catches made in April, roo miles from the nearest port, and when, for the offshore and inshore fish, the growth as calculated from the scales is compared, the agreement warrants the conclusion that the young fish have moved shoreward from the deeper waters. In good seasons this movement towards the shore coincides with increased landings of herrings.

Throughout June waves of migrating herrings come on to the grounds, and in the beginning of July the migrations are large enough to bring about a considerable increase in the fishery. These June and early July migrants have been found, off the Northumberland coast, to be marked with a comparatively small firstyear growth, as determined from the scales, which, for the most part, show three winter rings. Recovering spents from spring spawning shoals are found among catches of young developing herrings, but after the beginning of July they disappear, or the numbers found are insignificant.

Herrings with three winter rings and with a comparatively larger first-year growth than the June fish invade the grounds during July and August and give the high catches which are taken in these months in a successful fishery. Towards the end of August and the beginning of September shoals of larger and older herrings appear. They are full fish with their reproductive organs developed, and they, together with the young herrings sufficiently developed, form autumn spawning shoals. After spawning they disappear quickly and only young fish are to be caught.

The summer fishery of the east coast, the Shetlands excluded, is one which depends chiefly on young fish, and samples examined from Wick to Scarborough have been found to contain from 50 to 70 per cent. of fish with three winter rings on their scales. Fish of this age, therefore, determine the productivity of the fishery.

In September herrings are caught in the vicinity of the Dogger Bank by Dutch luggers and by trawlers, off Scarborough and Grimsby by drift-nets. Some of these fish are autumn spawners, but some, especially those caught by trawlers, are spring spawners, which now make their reappearance in great numbers. An examination of catches made on these grounds points to the herrings coming from the north-east to the southwestern end of the Dogger Bank and then moving in a south-westerly direction to the Grimsby grounds.

The East Anglian harvest begins in September and continues to the beginning of December. In the early part of the fishery many of the catches are landed from the grounds off Grimsby and it is not until October that the large fleets concentrate off Yarmouth and Lowestoft. This fishery is essentially one for full 